\title{
Patterns and Spatial Organisation: Culture, History and Future Perspectives
}

\author{
Yannis Zavoleas ${ }^{1,2}$. Mark Taylor ${ }^{3}$
}

Accepted: 22 January 2021 / Published online: 13 February 2021

(c) Kim Williams Books, Turin 2021

\begin{abstract}
Nexus Network Journal guest editors Yannis Zavoleas and Mark Taylor introduce the papers in vol 23 no. 1, a special issue dedicated to patterns and spatial organisation. In this introduction they discuss the role of patterns in architecture, from the early uses through to recent applications and future possibilities. Especially since the integration of computers and computing into design practice, a comparative discourse on the eras before-and-after their arrival seems imperative, so that the qualities of the past are selectively tailored to support those of the future. To that aim, the theme of this issue provides a vehicle to address many of architecture's key challenges from both a humanistic perspective and a technical/technological one.
\end{abstract}

Keywords Patterns $\cdot$ Spatial organisation $\cdot$ Systemic thinking $\cdot$ Cross-disciplinary thinking $\cdot$ Generative process $\cdot$ Dynamic patterns $\cdot$ Complexity $\cdot$ Semantic network method

This special issue on patterns introduces 13 research papers selected to represent the various aspects of that theme and refereed and revised by experts. The works expand upon a range of topics, so that the reader is prompted towards cross-disciplinary and cross-theoretical links between themes that otherwise appear as separate. A related call was announced in 2019 that outlined patterns' long association with architecture, from early uses as geometric ordering systems and the recognition of patterns within patterns, spanning across Greek, Roman, Islamic, and many other distinct architectural eras. That call further pointed out the more recent instances of

Yannis Zavoleas

y.zavoleas@uoi.gr; y.zavoleas@unsw.edu.au

Mark Taylor

marktaylor@swin.edu.au

1 University of Ioannina, Ioannina, Greece

2 University of New South Wales, Sydney, Australia

3 Swinburne University of Technology, Hawthorn, Australia

Birkhäuser 
aperiodic packing recognising non-repetitive and at times unpredictable geometry, conveyed through two-dimensional tiling or three-dimensional polyhedra, in a sense shifting the diagram from the seemingly static to a responsive and dynamic system. In view of those advances, it was suggested to revisit the legacy of mathematics into spatial organisation and structural resolution from the perspective of an architectural history and theory, relative to future models and simulations.

The main focus of this present issue has therefore been to link advanced technological endeavours and technology's more recent intellectual implications with core discourse in architecture and urban design (urbanism) developed since early Twentieth century. The guest-editors hope to enable a discussion on different logics of spatial development, and on how various periods utilised available methods and technologies towards different purposes. Several papers have been included drawing links between systemic thinking related to cybernetics, or bioinspired models and topological geometry when formulated as a holistic design approach applied across various scales. The works discuss themes on data collection and analytics, extended modelling, resource management, schema development, and form/material use. Much of this has been employed by the contemporary avant-garde, as a comprehensive framework to construct both ideology and relevance to the field for architectural, urban and landscape scenarios. With this selection of articles, it has specifically been attempted to weave connections between original practices and other cross-disciplinary areas of scientific and theoretical discourse.

The above themes have acquired new meaning through the work on patterns developed for this specific issue by a group of academics. Yannis Zavoleas and Mark Taylor's paper "New Relevancies Between Cultural Context and Spatial Discourse: An Evolution of Patterns through Methods, Models and Techniques" traces the use of patterns as ordering systems corresponding to different technologies and models of thought. The geometric patterns of modernism have gradually given way to ones being adaptive to different scenarios. The discourse has been reignited recently with the use of patterns as real-time translations of vast datasets. John Charles Driscoll's article "Fractal Patterns as Fitness Criteria in Genetic Algorithms Applied as a Desig $\mathrm{n}$ Tool in Architecture" introduces into the current computational context concepts, uses and applications of notions and problematics such as fractal arrangements. In this paper, fractals are explored as a bridge between nature, the built-environment and computational mechanics.

A set of articles delve into measuring performance, optimisation and expanding applications of patterns with generative techniques. The paper "Combinatorial Possibilities and Enumerations for Housing Designs: Gregory Ain's Mar Vista Tract (1946-1948)" by Jin-Ho Park and Sejung Young, introduces a formal method with reference to point group symmetry in the analysis, arrangement and construction of housing units. Wenzhi Huangfu, Michael J. Ostwald and Thomas Chung's paper "Visual-Asymmetry in Adolf Loos's Moller House: A Computational Analysis Testing Propositions about Performance and Spectation" revisits the famous early modernist design and uses isovist and visibility graph pattern analysis techniques (ASVI) to provide an updated understanding of the asymmetrical spatial relationships developed in the interior. 
Orkan Zeynel Güzelci, Sema Alaçam, Asena Kumsal Şen Bayram and Igor Lacroix's paper "Measuring the Entropy of Sinan's Muqarnas Patterns" presents a novel computational model to measure the entropy value of two-dimensional geometric patterns. The researchers have tested the proposed model in the muqarnas patterns in the portal of 12 mosques by architect Sinan during Sixteenth century. The findings suggest that the automated calculations are applicable to the selected sample group, with the potential to be adapted into different contexts. Graziano Mario Valenti and Chadi El Khoury's research paper "Let's Join: A Pavilion Inspi red by the Weaire and Phelan Space Tessellation" discusses the challenges and the results of modular tessellation techniques producing a surface of polyhedral faces that adapts dynamically to social activities hosted in a pavilion. Generative pattern making produces an agile modular system that is implemented and controlled using parametric and procedural models.

The next group of papers presents different historical approaches on dynamic patterns of cross-disciplinary historical referencing. Georgios-Spyridon Athanasopoulos' article "Emerging Spanning Trees in the Work of Candilis-Josic -Woods" discusses adaptations of natural patterns into spatial organising structures. Graph theory is combined with key late-modernist strategies of Team X and CIAM critics, also geometry and computational design methods for city planning and design reinforcing current strategic concepts on complex urban networks. Diana Cristobal Olave in "The Computer-Aided Rough Patterns of Christopher Alexa nde" analyses the pioneer's combined use of mathematical graphs and hand-made diagrams and argues that such affinities marked the introduction of algorithmic roughness with computational thinking in architecture. The employed techniques of transcription and calculation reveal the progressive abolishment of determinacy, favouring instead rough diagrams and mathematical graphs to serialise variation and to provide indeterminacy and contingency within a set of rules. Veronica Bastai, Andrea Cavani, Francesco Gherardini and Giulio Orsini's work "The Strut tura Reticolare Acentrata, a Design Strategy for Spatial Organization" uncovers adaptive design applications supported by natural concepts and tools. The Noncentred Reticular Structure is a modular pattern for spatial and urban planning focusing on trees developed by Cesare Leonardi. A set of irregular polygons defines areas that may belong to humans, plants and animals. The proposed network may evolve in space and time, combining biomimicry and geometry, whereas through metamorphosis and deformations, it adapts to different contexts and functional design elements. Lefteris Theodosis' article "Systemic Methods and Large-Scale Models in Ekistics" draws upon a geo-political understanding of the deeper consequences of complexity in urbanism and planning. The post-war era pioneering work of the architect-planner Constantinos A. Doxiadis and his concept of Ekistics are used as historical background for assessing the theoretical underpinnings and scope of contemporary smart city models.

Another set of papers proposes mathematical and structural analysis informing new design strategies. Ran Xiao's paper "Comparing and Clustering Residential Layouts using a Novel Measure of Grating Difference" employs methods by which to summarise generic patterns from a whole body of exemplary designs, which may be then critically assessed and applied to future projects. It applies clustering to a 
dataset of residential layouts to reveal underlying typological affinities. By extending such explorations, this paper suggests dynamic applications analogous to machine learning to inform new typologies and generic schemes. Jun Dong, Yongjin Wang and Rongrong Yu present the paper "Application of the Semantic Network Method to Sightline Compensation Analysis of the Humble Administrator's Garden", focusing on semantic network patterns supporting high levels of spatial complexity and variety applied on landscaped architectural space. Their study sets a new method for analysing and understanding contemporary landscape architecture intended for practitioners, students and educators. Jannis Athanasopoulos' article "Patterns and Tonalities in Architecture and Music" draws profound analogies between musical and spatial patterns around the key musical terms of tonality and atonality. Such cross-disciplinary analogies both technical and conceptual ones are proposed to enrich the understanding of regulating mechanisms in the two creative fields, and moreover to describe the means by which the architect may promote certain kind of interaction between the structured environment and its user.

As a final note to this editorial introduction, the guest-editors wish to thank the authors, also the several reviewers and the various roles at Nexus group that have contributed to this special issue. It is an honour that you have all trusted your work with us. We hope that this present issue is mostly rewarding and its themes versatile and inspiring to all who read it!

Publisher's Note Springer Nature remains neutral with regard to jurisdictional claims in published maps and institutional affiliations.

Yannis Zavoleas is Associate Professor at University of Ioannina, Ioannina, Greece, Adjunct Senior Lecturer at University of New South Wales, Sydney, Australia, and co-founder of Ctrl_Space Lab, Athens, Greece. His work explores the idea of "tools for thought" to expand creativity, by also introducing themes from biology, media and philosophy into architectural design. He has received awards in design, paper writing, fabrication and interactive storytelling. His funded research "Bio-shelters: Designing reef habitats at Sydney Harbour" combines data-driven design approaches with dynamic simulation to tackle environmental problems. Co-author (with Nicole Gardner and M. Hank Haeusler) of Computational Design: From Promise to Practice (2020); author of Machine and Network as Structural Models in Architecture (2013); co-editor (with Nikolas Patsavos) of Digital Materiality and the New Relation between Depth and Surface (2013).

Mark Taylor is Professor of Architecture at Swinburne University of Technology, Melbourne, Australia. His research on the interior has been widely published in journals and books, and he has taught both theory and design studio courses. He has held several nationally competitive grants and currently holds an Australian Research Council Discovery Grant to investigate Place and Parametricism. Taylor co-edited (with Julieanna Preston) the seminal publication Intimus: Interior Design Theory Reader (2006), edited the four-volume anthology Interior Design and Architecture: Critical and Primary Sources (2013), and co-edited, Designing the French Interior: The Modern Home and Mass Media (2015) and Flow: Interiors, Landscapes and Architecture in the Era of Liquid Modernity (2018). 